\title{
Copper(I) Hydride-Catalyzed Asymmetric
}

Hydrosilylation of Heteroaromatic Ketones

Bruce H. Lipshutz, ${ }^{*}$ Asher Lower, and Kevin Noson

Department of Chemistry \& Biochemistry, University of California, Santa Barbara, CA 93106 USA

Supporting Information 
Following the representative procedure provided in reference 14, the following amounts of reagents were used for entries 2-8 in Table 1. Characterization data is provided for new compounds.

Entry 2. 3-Acetylpyridine. 3-Acetylpyridine ( $0.50 \mathrm{~mL}, 4.50 \mathrm{mmol}), \mathrm{CuCl}(4.5 \mathrm{mg}, 0.045 \mathrm{mmol})$, $\mathrm{NaO}-t$-Bu (4.4 mg, $0.045 \mathrm{mmol}),(-)$-DTBM-SEGPHOS (2.7 mg, 2.25 x $\left.10^{-3} \mathrm{mmol}\right)$, PMHS (1.10 mL, $18.0 \mathrm{mmol})$, toluene $(9.0 \mathrm{~mL})$. Known compound; spectral data can be obtained from the Supporting Information within reference 1.1

Entry 3. 4-Acetylpyridine. 4-Acetylpyridine (1.00 mL, $9.04 \mathrm{mmol}), \mathrm{CuCl}(18.5 \mathrm{mg}, 0.187 \mathrm{mmol})$, $\mathrm{NaO}-t$-Bu (18.3 mg, $0.190 \mathrm{mmol})$, (-)-DTBM-SEGPHOS (6.0 mg, $\left.5.1 \times 10^{-3} \mathrm{mmol}\right)$, PMHS (3.0 mL, $45.5 \mathrm{mmol})$, toluene $(15.7 \mathrm{~mL})$, THF $(2.3 \mathrm{~mL})$. Known compound; spectral data can be obtained from the Supporting Information within reference $1 .{ }^{1}$

Entry 4. 2-Acetylfuran. 2-Acetylfuran $(630 \mathrm{mg}, 5.72 \mathrm{mmol}), \mathrm{CuCl}(5.7 \mathrm{mg}, 0.057 \mathrm{mmol}), \mathrm{NaO}-t$ $\mathrm{Bu}(5.5 \mathrm{mg}, 0.057 \mathrm{mmol})$, (-)-DTBM-SEGPHOS (3.4 mg, $\left.2.58 \times 10^{-3} \mathrm{mmol}\right)$, PMHS (1.50 mL, 22.9 $\mathrm{mmol})$, toluene $(5.75 \mathrm{~mL})$. Known compound; spectral data can be obtained from the Supporting Information within reference $1{ }^{1}$

Entry 5. 1-Furan-2-ylheptan-1-one. 1-Furan-2-yl-heptan-1-one (0.82 mL, $5.00 \mathrm{mmol}), \mathrm{CuCl}(4.95$ $\mathrm{mg}, 0.050 \mathrm{mmol}), \mathrm{NaO}-t$-Bu $(4.80 \mathrm{mg}, 0.050 \mathrm{mmol})$, (-)-DTBM-SEGPHOS (2.95 mg, $2.50 \times 10^{-3}$ $\mathrm{mmol})$, PMHS (1.30 mL, $20.0 \mathrm{mmol})$, toluene $(5.00 \mathrm{~mL})$. Known compound; spectral data can be obtained from the Supporting Information within reference $2{ }^{2}$

Entry 6. 2-Acetylthiazole. 2-Acetylthiazole ( $0.630 \mathrm{~mL}, 6.08 \mathrm{mmol}), \mathrm{CuCl}(6.02 \mathrm{mg}, 0.0608 \mathrm{mmol})$, $\mathrm{NaO}-t$-Bu (5.84 mg, $0.0608 \mathrm{mmol})$, (-)-DTBM-SEGPHOS (3.59 mg, $\left.3.04 \mathrm{x} 10^{-4} \mathrm{mmol}\right)$, PMHS (1.58 $\mathrm{mL}, 24.3 \mathrm{mmol})$, toluene $(6.00 \mathrm{~mL})$. Known compound; spectral data can be obtained from the Supporting Information within reference $1 .^{1}$

Entry 7. 2,5-Dimethyl-4-acetylthiazole. 2,5-Dimethyl-4-acetylthiazole $(0.20 \mathrm{~mL}, 1.48 \mathrm{mmol})$,

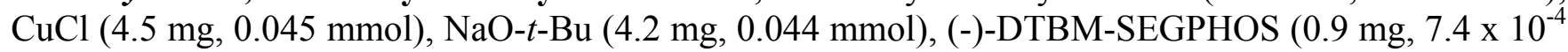
$\mathrm{mmol})$, PMHS $(0.19 \mathrm{~mL}, 3.0 \mathrm{mmol})$, toluene $(1.50 \mathrm{~mL})$. Known compound; spectral data can be obtained from the Supporting Information within reference $3 .^{3}$

Entry 8. 1-(5-Methylisoxazol-3-yl)-pentan-1-one. 1-(5-Methylisoxazol-3-yl)-pentan-1-one (57 mg, $0.34 \mathrm{mmol}), \mathrm{CuCl}(0.34 \mathrm{mg}, 0.0034 \mathrm{mmol}), \mathrm{NaO}-t-\mathrm{Bu}(0.33 \mathrm{mg}, 0.0034 \mathrm{mmol}),(-)-D T B M-S E G P H O S$ $\left(0.2 \mathrm{mg}, 1.7 \times 10^{-4} \mathrm{mmol}\right)$, PMHS $(0.09 \mathrm{~mL}, 1.36 \mathrm{mmol})$, toluene $(0.34 \mathrm{~mL})$. Spectral data was obtained for the acetate derivative. Colorless oil. $300 \mathrm{MHz}{ }^{1} \mathrm{H} \mathrm{NMR}\left(\mathrm{CDCl}_{3}\right) \delta=0.89(\mathrm{t}, J=5.4 \mathrm{~Hz}, 3 \mathrm{H}$, methyl), $1.33(\mathrm{~m}, 2 \mathrm{H}$, methylene), $2.10(\mathrm{~m}, 2 \mathrm{H}$, methylene), $2.11(\mathrm{~s}, 3 \mathrm{H}$, acetate methyl), $2.14(\mathrm{~m}, 2 \mathrm{H}$, methylene), 2.20 (s, 3H, oxazole methyl), 5.86 (t, $J=4.8 \mathrm{~Hz}, 1 \mathrm{H}$, methine), 5.95 (s, 1H, oxazole ring); IR $\left(\mathrm{cm}^{-1}\right)$ 2253, 1711, 1647, 1412, 909; exact mass calcd for $\mathrm{C}_{11} \mathrm{H}_{17} \mathrm{NO}_{3}$ 211.1208, found: 211.1207.

\footnotetext{
${ }^{1}$ Ohkuma, T.; Koizumi, M.; Noyori, R. Org. Lett. 2000, 12, 1749.

${ }^{2}$ Maurizio, D.; Rocco, R.; Gianfranco R. Eur. J. Org. Chem. 2000, 19, 3265.

${ }^{3}$ Fantin, G.; Fogagnolo, M.; Medici, A.; Pedrini, P.; Poli, S. Tetrahedron: Asymmetry 1993, 4, 1607.
} 